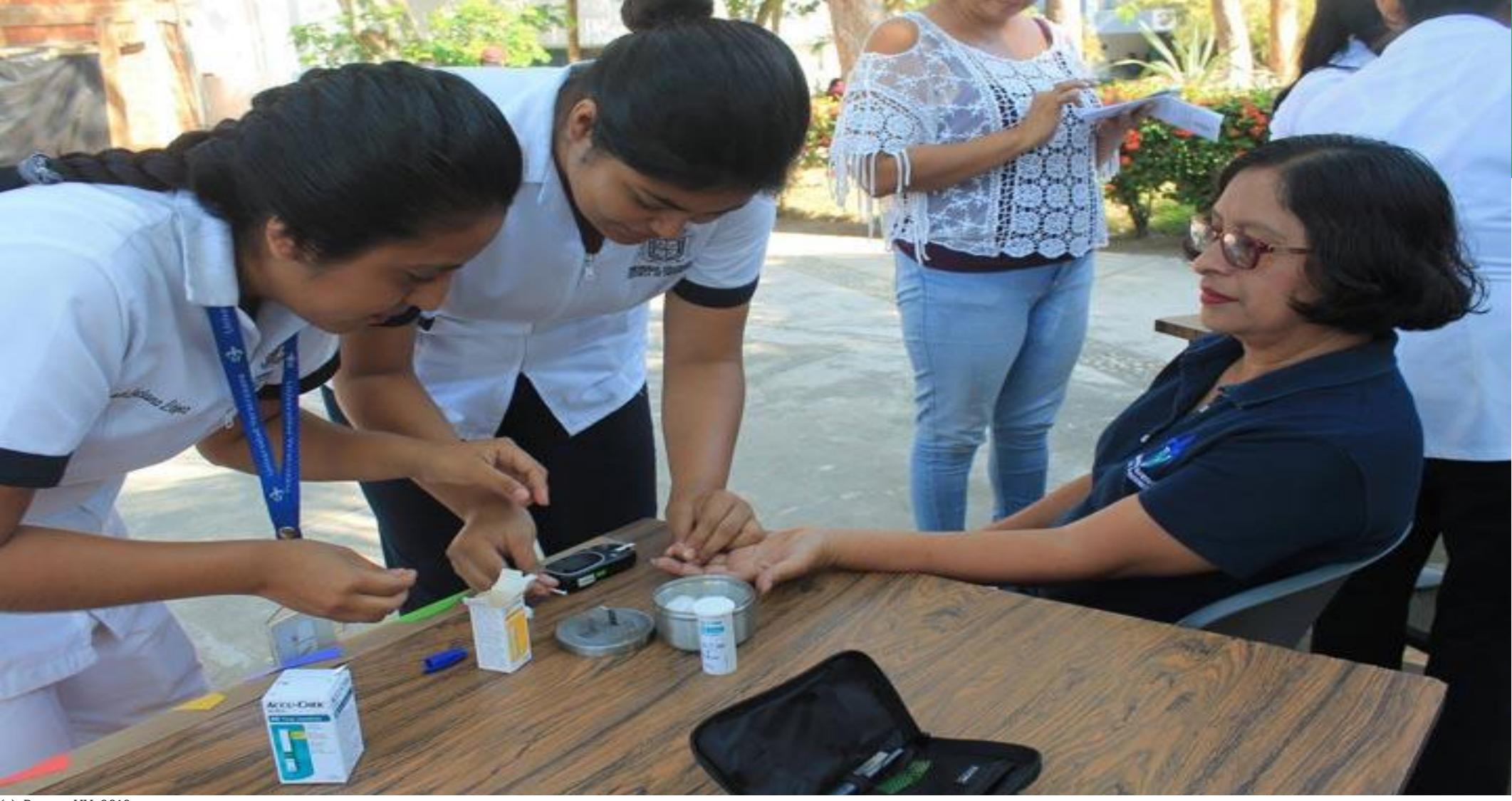

\title{
Apego al tratamiento en pacientes diabéticos y sus creencias
}

Guadalupe Camarillo Guzmán - Rocío López Lara - Jaime Morales Romero - Carlos Flores Pérez - Verónica Patricia Demenegui Marini - Sergio Luis García Gallegos ${ }^{1}$

RESUMEN: Introducción. El uso de terapias alternas y complementarias en enfermedades crónicas como la diabetes es una práctica relevante hoy en día, se busca tratar de comprender el trasfondo de los motivos por los cuales se recurre a ella. Objetivo. Explorar las razones por las cuales las personas recurren a tratamientos alternativos. Metodología. Estudio cualitativo, se realizaron entrevistas semi-estructuradas. El discurso transcrito se codificó y se realizó análisis de contenido temático. Resultados._La concepción sobre la enfermedad varía, aunque predomina un significado de gravedad y muerte. Las terapias alternas son utilizadas de forma complementaria para controlar la diabetes y retrasar las

\footnotetext{
${ }^{1}$ Guadalupe Camarillo Guzmán, Facultad de Enfermería, Universidad Veracruzana. Autor correspondiente. contacto: gcamarillo@uv.mx; Rocío Lopez Lara, Instituto de Salud Pública, Universidad Veracruzana; Jaime Morales Romero; Carlos Flores Pérez; Verónica Patricia Demenegui Marini; Sergio Luis García Gallegos
} 
complicaciones, así como los malestares físicos que provoca el medicamento. Conclusiones. Los pacientes recurren al uso de terapias alternas por el temor a las complicaciones de la enfermedad, los efectos secundarios de los medicamentos y la falta de apoyo sociofamiliar para seguir el tratamiento farmacológico.

Palabras clave: Diabetes, Tratamiento, terapias alternas y complementarias

Abstract: The use of alternative and complementary therapies in chronic diseases such as diabetes is a relevant practice nowadays. It seeks to understand the background of the reasons why it is used. Objective. Explore the reasons why people turn to alternative treatments. Methodology. Qualitative study, semi-structured interviews were conducted. The transcribed discourse was codified, and the thematic content analysis was carried out. Results. The conception about the disease varies although a meaning of seriousness and death predominates. Alternate therapies are used in a complementary way to control diabetes and delay complications as well as the physical discomfort caused by the medication. Conclusions. Patients resort to the use of alternative therapies because of the fear of complications from the disease, the side effects from the medications and the lack of socio-family support to follow the pharmacological treatment.

Key words: Diabetes, Treatment, Alternative and Complementary Therapies.

\section{Introducción}

L

a diabetes mellitus tipo 2 y sus complicaciones constituyen un importante problema en salud pública. El tratamiento forma parte importante en el control de esta. Sin embargo, las fallas en las estrategias para el control y tratamiento de la diabetes dejan entrever las deficiencias de un sistema de salud incapaz de afrontar de manera integral dicha patología (Organización Mundial de la Salud, 2017). La Diabetes ha ido en aumento de manera desproporcionada en varios países, ocupando_los primeros lugares como causa de mortalidad. Para el año 1980 se estimaba un total de 108 millones de personas con diabetes en el mundo, cifra que para el año 2014 ascendió a 422 millones de personas con diabetes mellitus (Organización Mundial de la Salud, 2017).

Según los perfiles por países de la OPS, el 14\% de las muertes en México se deben a la Diabetes Mellitus tipo 2, solo por debajo de las enfermedades cardiovasculares. De igual manera, la prevalencia de diabetes en México por sexo es de 9.7\% para hombres y 11.0\% para mujeres (Plataforma de información en Salud de las Américas, 2017). 
La diabetes mellitus está considerada como uno de los principales problemas de salud pública a nivel mundial. Existen 285 millones de personas con diabetes en todo el mundo, la prevalencia de diabetes para el año 2010 fue de 6.6\% y se espera que para el 2030 aumente a 7.8\% (Adeghate, E., 2006).

La búsqueda de tratamiento complementario obedece al grado de severidad percibido por el paciente, en la medida en que se sienta agravado por su padecimiento, será la búsqueda intencionada de remedios que logren mitigar su "malestar", e incluso la eliminación del mismo (Moreno, E., 2003)

\section{Contenido}

\section{La diabetes mellitus tipo 2 y el tratamiento farmacológico}

Según la Organización Mundial de la Salud (OMS), la diabetes mellitus tipo 2 se origina cuando el organismo es incapaz de utilizar eficazmente la insulina producida por el páncreas, siendo esto consecuencia del exceso de peso o inactividad física. La insulina es la hormona encargada de regular los niveles de glucosa en la sangre; el efecto de la diabetes no controlada es la hiperglucemia que, posteriormente trae consecuencias graves como daño a órganos y sistemas, principalmente los sanguíneos (Organización Mundial de la Salud, 2017).

\section{Tratamiento}

El tratamiento y control de la diabetes está encaminado a prevenir las complicaciones agudas y crónicas de la enfermedad, mejorar la calidad de vida y reducir la mortalidad atribuible a esta enfermedad o a sus complicaciones. El plan de manejo debe contemplar tratamiento farmacológico, no farmacológico, educación al paciente, automonitoreo y vigilancia (NOM-015-SSA2-1994).

El tratamiento farmacológico considera dos opciones: los hipoglucemiantes orales y la administración de insulina. Debe ser prescrito cuidadosamente bajo vigilancia médica, especialmente cuando se presenta hiperglucemia sintomática, esto con la finalidad de llegar a metas propuestas con anterioridad, una de ellas, niveles de glucosa normales 60 a 110 mg/dL (NOM-015-SSA2-1994).

Cuando los pacientes no logran controlarse con la dieta y el ejercicio físico, se recurre a los fármacos para disminuir los niveles de glucosa en la sangre. Para que el médico tratante 
seleccione correctamente el tratamiento farmacológico debe contemplar ciertas características de los pacientes, entre ellas la edad, el estado cardiovascular, verificar si existe insuficiencia hepática o renal, embarazo o lactancia, alergia a las sulfas, entre otros (Zarate A., 1990).

A diferencia del tratamiento farmacológico, el control de la diabetes desde la perspectiva no farmacológica considera acciones que tienen que ver con el auto cuidado del paciente, tales como el plan de alimentación y el ejercicio físico. La atención no farmacológica está relacionada con la educación para la salud que la persona con diabetes recibe (NOM-015SSA2-1994).

\section{Uso de terapias alternativas y complementarias}

De acuerdo con la Organización Mundial de la Salud (OMS), la medicina tradicional es la suma completa de conocimientos, técnicas y prácticas fundamentadas en las teorías, creencias y experiencias propias de diferentes culturas. Su uso es para mantener la salud, prevenir, diagnosticar, mejorar o tratar trastornos físicos y mentales. Cuando la medicina tradicional es adoptada por otras poblaciones se le llama también alternativa o complementaria (Organización Mundial de la Salud, 2014).

En general, se conoce como medicina alternativa a una gran variedad de terapias y prácticas que varían entre países y entre regiones y que han sido utilizadas desde hace miles de años, entre las poblaciones indígenas y de la cultura oriental, principalmente; se identifican como medicina o terapias alternativas: acupuntura, herbolaria, homeopatía, quiropráctica, fitoterapia, reflexología, masajes, kinesiología, aromaterapia, consumo de suplementos nutricionales, curanderos espirituales, entre otros. Se les ha llamado alternativas pues representan "otro" medio para atender la enfermedad y conservar la salud. En los últimos años también se les conocen como "complementarias", ya que son usadas en conjunto con los tratamientos médicos convencionales y no de forma sustitutiva (Aedo, F., 2000).

Las enfermedades crónicas, entre ellas la diabetes, están relacionadas con una mayor incidencia en el uso de medicina complementaria, siendo las creencias de los pacientes la principal razón por la cual acuden a ella, con la intención de mejorar su calidad de vida. Otro factor que interviene en esta decisión es evitar los métodos invasivos y los efectos secundarios de los medicamentos, así como la insatisfacción del trabajo médico y las motivaciones filosóficas y culturales (Aedo, F., 2000). 
En muchos casos el costo de los medicamentos y la dificultad para manejar la dieta, cuando no se recibe asesoría nutricional adecuada, representan factores para que el paciente diabético abandone el tratamiento médico, dando pauta a una descompensación que puede llegar a ocasionar complicaciones severas. Todo ello influye para que el paciente busque alternativas terapéuticas que le resulten económicas y de fácil manejo, además de que formen parte de sus conocimientos y referentes socioculturales (Saucedo, M., 2006).

\section{Proceso salud-enfermedad desde la Antropología Médica}

En relación al proceso salud-enfermedad-atención, la antropología médica pone de manifiesto una perspectiva diferencial a la posición de las teorías biomédicas que explican el desarrollo de la enfermedad en términos biológicos y anatómicos. En el caso del apego y la adherencia al tratamiento, busca comprender cómo intervienen diversos factores en un adecuado control metabólico. Según la antropología médica, la concepción que una persona tiene sobre la salud-enfermedad va de la mano con su contexto social e individual; su actuar ante la enfermedad depende de las ideas que ha construido en torno a ella, así como de su propia historia con respecto a su padecer (Moreno, L., 2007).

Para el modelo antropológico, el padecimiento está supeditado por la forma en que el individuo percibe y expresa el proceso de enfermar, antecediendo el padecimiento al malestar, mismo que el individuo asocia con algo biológico que sabe que no está funcionando bien. Entendido así, la diabetes mellitus es un "padecimiento" que entremezcla diversidad de elementos que más allá de constituir una idea aislada de la diabetes, la hacen ver como una enfermedad que trae consigo malestares que el individuo expresa mediante su capacidad de resolución o en la búsqueda de atención (Moreno, L., 2007).

\section{El modelo de creencias en salud}

Aproximación teórica que explica el problema del cumplimiento terapéutico por parte de los pacientes. Manifiesta que las creencias y expectativas influyen en la conducta y señala que el seguimiento de las recomendaciones dependerá de factores como: el grado de amenaza de la sintomatología, determinado por el nivel de susceptibilidad o vulnerabilidad percibido por el individuo ante la enfermedad, así como por la aceptación del diagnóstico; el grado de severidad de la enfermedad y sus consecuencias al no atenderla; la percepción de los 
beneficios y costos que traerá consigo el tratamiento y el grado de motivación hacia la salud, relacionado con la preocupación del paciente acerca de mejorar su salud y con su intención de cambio de conductas (D Anello, S., 2009).

Este modelo plantea que el apego al tratamiento requiere la adopción de conductas por parte de las personas, dicho comportamiento está basado en un análisis subjetivo y según el cual estiman el costo-beneficio de cada conducta; los pensamientos de las personas sobre cada conducta posible (cuidarse, seguir un tratamiento, abandonarlo, acudir a tratamientos alternativos o complementarios) son patrones arraigados, producto de su cognición individual y del sentido social que para su grupo tiene tanto la enfermedad como la forma de curarse; por lo tanto, las creencias son patrones culturales que tienen un efecto directo en el mejoramiento del estado de la persona, en tanto la persona asocie las conductas de cuidado a una utilidad mayor esperada (Ehrenzweig, Y., 2007)

Estas explicaciones socio-antropológicas guardan relación con una conceptualización de la medicina alternativa o terapia alterna como una práctica derivada de la cultura de cada país o región para tratar las enfermedades que aquejan a la población. Las terapias alternas son cada vez más variadas y se caracterizan por su origen autóctono y su transmisión de generación en generación (Sistemas de salud tradicionales en América Latina y el Caribe, 2017).

\section{Material y Método. Diseño del estudio}

Investigación de naturaleza cualitativa con alcance descriptivo, pues se realizó una lectura anecdótica, de tipo etnográfica del fenómeno y sus características. En estos estudios el discurso de los informantes es organizado temáticamente (Mercado, F., 2007).

\section{Tipo de muestreo}

Muestreo intencional de casos-tipo. Es una estrategia de muestreo no probabilística, basada en la representatividad socio-estructural de los participantes, para la cual se delimita previamente el grupo social representado y los atributos que lo caracterizan (Hernández, R., 2006; Flick, U., 2004). 


\section{Tipos sociales}

Pacientes con diagnóstico de diabetes mellitus, adscritos a la Unidad de Medicina Familiar $\mathrm{N}^{\circ} 66$ del IMSS en la ciudad de Xalapa, Veracruz, que se hayan identificado por el uso de terapias alternas. Para asegurar la riqueza y calidad del discurso, se buscó que la muestra guardara un equilibrio entre homogeneidad y heterogeneidad (Navarrete, J., 2000).

\section{Tamaño de la muestra}

El muestreo cualitativo se perfila progresivamente, en cuanto avanza el examen de los casos, esto significa que el tamaño de la muestra no es definido a priori, sino que va emergiendo durante el desarrollo de la investigación, en la búsqueda del punto de saturación de los datos. El punto de saturación se originó en la décima entrevista, por lo que la muestra estuvo constituida por 10 informantes.

\section{Técnica para la obtención de información}

\section{Entrevista semi-estructurada}

Técnica utilizada para conocer la opinión que la gente tiene respecto a algún evento o fenómeno, en este caso de salud, no necesariamente sigue una secuencia previamente establecida, dicho de otra manera, la entrevista está condicionada a las respuestas que vaya aportando el informante.

\section{Plan para el procesamiento de la información}

El trabajo analítico consistió en proponer vínculos entre los datos; lo que le dio soporte al trabajo es la manera cómo se usaron los códigos y los conceptos. Los datos obtenidos y su correcto uso y codificación permitieron generar ideas en torno al tema de estudio. La información que se obtuvo en el trabajo de campo fue codificada y analizada partiendo de grandes ejes y categorías que se fueron afinando durante el análisis. 
Tabla 1.

Ejes de análisis y categorías conceptuales para el estudio

\begin{tabular}{|l|l|}
\hline Ejes de análisis & Categorías conceptuales \\
\hline La enfermedad & $\begin{array}{l}\text { Concepto de la enfermedad } \\
\text { Origen/causalidad de la enfermedad } \\
\text { Diagnóstico de la enfermedad } \\
\text { Cronicidad y efectos de la enfermedad } \\
\text { Curación de la enfermedad } \\
\text { Control de la enfermedad }\end{array}$ \\
\hline El tratamiento & $\begin{array}{l}\text { Tratamiento farmacológico y no farmacológico } \\
\text { Condiciones para el apego }\end{array}$ \\
\hline Medicina alternativa & $\begin{array}{l}\text { Origen del uso } \\
\text { Efectos/beneficios sobre la enfermedad } \\
\text { Complementariedad o alternativa al tratamiento } \\
\text { farmacológico }\end{array}$ \\
\hline
\end{tabular}

Fuente: Elaboración propia

\section{Resultados}

Este estudio estuvo compuesto por personas de edad mayor a los 50 años, la_escolaridad predominante es de primaria. El tiempo que tiene con la enfermedad va de los 6 a los 25 años. La mayoría de los informantes dijo ser casado/a. Las creencias religiosas son múltiples, siendo más común la religión católica. En todos los casos, los sujetos entrevistados referían hacer uso de algún método de cuidado complementario.

\section{Concepto de la Enfermedad}

El concepto que los sujetos entrevistados tienen sobre la enfermedad es variado; están aquellos que han sentido malestar general desde el diagnostico confirmatorio, por ende, la conciben como un fenómeno patológico que paulatinamente deteriora al organismo al mismo tiempo que las funciones esenciales del mismo. Contrario a lo anterior, sobresale la 


\section{Artículo - Ciencias de la Salud}

idea de que éste fenómeno patológico no es grave, consecuencia de ello son actitudes poco saludables y desapego al tratamiento farmacológico.

\section{Cronicidad y efectos de la enfermedad}

La diabetes es concebida como una enfermedad común, que "todo el mundo la tiene", y que por ello no es necesario cambiar el estilo de vida, esto lleva a pensar que padecer diabetes es algo inevitable y que nada_impedirá que se desarrolle la enfermedad. Deben ser consideradas las repercusiones psicológicas, ya que ocupan un campo amplio en el padecimiento de la diabetes. Expresiones de debilidad y “desguance” están acompañadas de síntomas de depresión y de angustia. Los efectos psicológicos acarrean comportamientos poco favorables que disminuyen la calidad de vida en los pacientes diabéticos. Reyna:

Físicamente [sic] y psicológicamente, te va consumiendo porque se siente algo por dentro, yo, yoooo... a pesar de que hago deporte, y de que trato que,... que me voy nutriendo, porque me tomo mi malteada temprano que está cargada de nutrientes, bueno, la otra que son puros multivitamínicos, entonces este... a pesar de que me sigo cuidando, en el aspecto de no decaer, por dentro me siento, hay días que me siento débil, haz de cuenta que... de pronto, incluso no sé, por la misma depresión siento mi corazón muy débil, hay días que me siento tan casada, tan agotada, tan sin ganas a veces de nada, que y a pesar de que son días que no estoy en depresión...

\section{Diagnóstico y origen de la enfermedad}

Sensación de tristeza, de decepción y de incertidumbre predominan cuando los pacientes señalan el momento en que fueron diagnosticados. El impacto de saberse enfermo viene acompañado de sensaciones físicas inexplicables para el sujeto. Esto está asociado con la gravedad percibida por el paciente.

Se identifican dos concepciones distintas en cuanto al origen de la diabetes: la primera recae en el estilo de vida. Para ellos la enfermedad se deriva de una vida sedentaria, por ingerir alimentos altos en grasas y carbohidratos; algunos señalan que es una patología hereditaria. La otra conceptualización la concibe como un mal provocado externamente, en el que poco pudo hacer la persona para impedirlo; se le asocia a situaciones desfavorables (sustos, corajes, tristezas, preocupaciones) que generan una gran impresión a la que le sigue la aparición de la diabetes. 


\section{Control de la enfermedad}

Los pacientes mencionan que la diabetes es una enfermedad que no tiene cura, sólo se controla con la dieta, ejercicio y con el medicamento. El auto cuidado participa como elemento central el cual logra reducir los niveles de glucosa en sangre hasta un nivel ideal.

\section{Condiciones para el apego al tratamiento farmacológico}

El apego al tratamiento constituye una serie de acciones que tienen como meta principal mantener los niveles de glucosa en la sangre controlados. Las indicaciones terapéuticas que hace el médico familiar deben ser comprendidas y aceptadas, sin embargo, la idea de saber que la diabetes implica un tratamiento de por vida hace que los pacientes tomen el medicamento de forma mecánica, automática, sin tener una concepción clara de los beneficios que esta acción conlleva.

\section{Condiciones para la falta de apego}

Provocado por olvido o malestar que propicia el medicamento, y además por influencia social; el paciente manifiesta un miedo sobre las consecuencias de tomar el medicamento; temen padecer gastritis severa y, en ciertas ocasiones dañar el riñón por la cantidad de medicamento administrado. José:

Pues... es dejadez mía, no me la tomo porque me choco de tanta medicina y pienso eso porque a veces algunas personas me dicen: descansa unos dos tres días porque también mucha medicina te va a causar un día algún este... un tumor, un tumor de tanta medicina, y si es cierto, tienen razón, porque hay muchas personas que padecen de tumores por la... pues de tanta medicina que les cae pesado...

\section{Origen del uso de terapias alternas}

Concebir la medicina alternativa como algo natural fue un elemento esencial para empezar a utilizarla, dado que se supone que lo natural siempre hace bien o es bueno para el organismo. Bajo este razonamiento el paciente o sus propios familiares buscan el tratamiento natural para complementar las indicaciones médicas. 


\section{Efectos benéficos de la terapia complementaria}

La confianza que tienen sobre estas terapias está representada como una fuerza religiosa, una creencia que con sólo tenerse fervientemente logra disminuir los estragos de la enfermedad. Cuando se toma el té o el remedio alterno, se crea una imagen de que es algo que purifica y limpia el organismo. La diabetes es una enfermedad que contamina, y para ello se necesita limpiarse por dentro.

\section{Conclusiones}

Este estudio confirma en lo general, lo señalado por el abordaje teórico de la antropología médica_(Menéndez, E., 2002). y por el modelo de creencias en salud con relación a que el sujeto enfermo responde a su enfermedad y a las medidas de tratamiento en función de una compleja interacción de ideas, patrones culturales y percepciones sobre su nivel de vulnerabilidad ante la enfermedad (Ronny, A., 2006).

El discurso recuperado permite confirmar lo que el modelo de creencias en salud señala respecto a que quienes conciben que la diabetes es una enfermedad severa, capaz de generar síntomas y secuelas dramáticas, quienes han tenido experiencias con familiares diabéticos y, por lo tanto, se piensan o sienten en riesgo de padecerla, son más susceptibles a apegarse al tratamiento; quienes refieren una alta motivación hacia mantener un estado saludable, que les permita ser autónomos, independientes, activos laboralmente, buscan la sanación tanto con el tratamiento farmacológico como con las terapias alternativas, tipo tés naturales y productos bioenergéticos.

El peso de las creencias y las ideas propias en torno a la severidad de la enfermedad, al riesgo de los medicamentos o a los beneficios de un tratamiento alternativo se confirma en los casos donde los pacientes expresan sentirse bien y mantenerse saludables a partir de llevar su tratamiento médico en combinación con alguna terapia alternativa (Cereceros, O., 2009; Taddei, A.,1999).

La antropología médica señala que la base del apego está en la forma como la persona entiende la enfermedad y el tratamiento, elemento semejante a las razones para acudir a terapias alternas, que tienen un enfoque más holístico, natural e incluso religioso; todo ello reitera la importancia de que los profesionales de la salud consideren los aspectos 
individuales y socioculturales del paciente, en la definición y ejecución de medidas para la atención y prevención de la enfermedad.

\section{Referencias}

Adeghate, E., Schattener P.,Dunn E. (2006). An up date on the etiology and epidemiology of diabetes mellitus. New York: AcadSci.

Aedo, F. (2000). La Medicina Complementaria en el Mundo. Revista Mexicana de Medicina Física y Rehabilitación, 2, pp.91-99.

Cereceros, O., Tortorielo, J. (2009). “Uso de plantas medicinales por pacientes con Diabetes Mellitus Tipo 2 en Morelos, México”. Boletín Latinoamericano y del Caribe de Plantas Medicinales y Aromáticas, 8, pp. 1-8.

D Anello, S. (2009). La relación Médico-Paciente y su influencia en la adherencia al tratamiento médico. MedULA, 18, pp.33-39.

Ehrenzweig, Y. (2007). Modelos de cognición social y adherencia terapéutica en pacientes con cáncer. Avances en Psicología Latinoamericana, 1, pp.7-21.

Flick, U. (2004). Introducción a la Investigación Cualitativa. Madrid, España: Morata.

Hernández, R., Fernández, C., Baptista, P. (2006). Metodología de la Investigación. México: McGraw-Hill.

Menéndez, E. (2002). El punto de vista del actor: Homogeneidad, diferencia e historicidad. Región y Sociedad, 20, p. 237.

Mercado, F. (2007). El caso de los servicios de salud. Investigación cualitativa en los servicios de salud Buenos Aires, Argentina.: Lugar Editorial. pp.109-135.

Moreno, E. (2003). El modelo de creencias en salud: revisión teórica, consideración crítica y propuesta alternativa, I: Hacia un análisis funcional de las creencias en salud. Internationl Journal of Psychology and Psychological Therapy, Vol. 3. Universidad de Almería, España.

Moreno, L. (2007). Reflexiones sobre el trayecto salud-padecimiento-enfermedad-atención. Una mirada socioantropológica. Salud Pública de México, 01, pp.63-70.

Navarrete, J. (2000). Investigaciones Sociales. México: pp. 166-176.

NOM-015-SSA2-1994. (1994). Norma Oficial Mexicana para la Prevención, Control y Tratamiento de la Diabetes Mellitus en la atención primaria a la salud. Recuperado de http://www.salud.gob.mx/unidades/cdi/nom/m015ssa24.html

Organización Mundial de la salud (2017). Sistemas de salud tradicionales en América Latina y el Caribe. Consultado el 10 de diciembre de 2017, disponible en: http://iris.paho.org/xmlui/handle/123456789/48336 


\section{Artículo - Ciencias de la Salud}

Organización Mundial de la Salud. (2014). Traditional Medicine Strategy 2014-2023. Consultado el 19 de febrero de 2018, Disponible en: https://www.who.int/traditionalcomplementary-integrative-medicine/en/

Organización Mundial de la Salud. (2017). Notas descriptivas. Consultado el 18 de junio de 2018, disponible en: http://www.who.int/es/news-room/fact-sheets/detail/diabetes

Plataforma de información en Salud de las Américas. (2017). Indicadores Básicos. Recuperado de http://www.paho.org/data/index.php/es/

Ronny, A. (2006). "Complementary and alternative medicine use and diabetes selfmanagement among rural older adults". Complement healt practics, 2, pp. 6-11.

Saucedo, M. (2006). La práctica de la medicina alternativa; una realidad en el paciente diabético en Hermosillo, Sonora. RESPYN. Revista Salud pública y nutrición, 7, p.4.

Taddei, A., Santillana, A., Romero, A., Romero, B. (1999). "Aceptación y uso de herbolaria en medicina familiar". Red de revistas científicas de América Latina, El Caribe, España y Portugal., 41, pp.216-220.

Zarate, A. (1990). Diabetes Mellitus: Bases para su tratamiento. México: Trillas. 